OPEN ACCESS

Edited by:

David Rodriguez-Lazaro, University of Burgos, Spain

Reviewed by:

Frieder Schaumburg, University Hospital Münster, Germany Marta Hernandez, Instituto Tecnológico Agrario de Castilla y León, Spain

*Correspondence: Luria L. Founou luriafounou@gmail.com

Specialty section: This article was submitted to Food Microbiology, a section of the journal Frontiers in Microbiology

Received: 17 November 2017 Accepted: 26 January 2018

Published: 09 February 2018

Citation: Founou LL, Founou RC, Allam M, Ismail A, Djoko CF and Essack SY (2018) Genome Sequencing of Extended-Spectrum $\beta$-Lactamase (ESBL)-Producing Klebsiella pneumoniae Isolated from Pigs and Abattoir Workers in Cameroon.

Front. Microbiol. 9:188.

doi: 10.3389/fmicb.2018.00188

\section{Genome Sequencing of Extended-Spectrum $\beta$-Lactamase (ESBL)-Producing Klebsiella pneumoniae Isolated from Pigs and Abattoir Workers in Cameroon}

\author{
Luria L. Founou 1,2*, Raspail C. Founou1,3, Mushal Allam4, Arshad Ismail4, \\ Cyrille F. Djoko ${ }^{5,6}$ and Sabiha Y. Essack ${ }^{1}$
}

\begin{abstract}
${ }^{1}$ Antimicrobial Research Unit, College of Health Sciences, University of KwaZulu-Natal, Durban, South Africa, ${ }^{2}$ Department of Food Safety and Environmental Microbiology, Centre of Expertise and Biological Diagnostic of Cameroon, Yaoundé, Cameroon, ${ }^{3}$ Department of Clinical Microbiology, Centre of Expertise and Biological Diagnostic of Cameroon, Yaoundé, Cameroon, ${ }^{4}$ Sequencing Core Facility, National Health Laboratory Service, Johannesburg, South Africa, ${ }^{5}$ Centre for Research and Doctoral Training in Life Science, Health and Environment, The Biotechnology Centre, University of Yaoundé I, Yaoundé, Cameroon, ${ }^{6}$ Metabiota Inc., Yaoundé, Cameroon
\end{abstract}

Background and objectives: Extended-spectrum $\beta$-lactamase (ESBL)-producing Klebsiella pneumoniae is a serious public health issue globally. In this study, the antibiotic resistance genes, virulence factors, mobile genetic elements, and genetic lineages of circulating ESBL-producing K. pneumoniae strains isolated from pigs and humans in Cameroonian abattoirs were investigated using whole genome sequencing (WGS), in order to ascertain zoonotic transmission (viz. from animals to humans and/or vice-versa) in the food chain.

Methods: During March-October 2016, 288 nasal and rectal pooled samples from 432 pigs as well as nasal and hand swabs from 82 humans were collected from Cameroon and South Africa. Seven ESBL-producing K. pneumoniae circulating in Cameroonian pig abattoirs were selected and their genomic DNA sequenced using an Illumina MiSeq platform. Generated reads were de novo assembled using the Qiagen CLC Genomics Workbench and SPAdes. The assembled contigs were annotated using RAST and antibiotic resistance genes, virulence factors, plasmids, and bacteriophages were identified with ResFinder, Virulence Finder, PlasmidFinder, and PHAST, respectively.

Results: ESBL-producing K. pneumoniae were detected in pigs (34/158; 21.52\%) and exposed workers $(8 / 71 ; 11.26 \%)$ in Cameroon only. The circulating K. pneumoniae strains were dominated principally by the sequence type (ST) 14 and 39. In addition, the "high-risk" ST307 clone and two novel STs assigned ST2958 and ST2959 were detected. Genomic analysis identified various antibiotic resistance genes associated with resistance to $\beta$-lactams, aminoglycosides, fluoroquinolones, macrolide, lincosamide and streptogramins, rifampicin, sulfonamides, trimethoprim, phenicols and tetracycline. None of the ESBL-producing $K$. pneumoniae harbored virulence genes. Intermingled K. pneumoniae populations were observed between pig- and human-source within and across abattoirs in the country. 
Conclusion: Our study shows that ESBL-producing $K$. pneumoniae is actively disseminating in pigs and occupationally exposed workers in Cameroonian pig abattoirs and is probably underestimated in the absence of molecular epidemiological studies. It suggests pigs, abattoir workers and food products as potential reservoirs and sources of zoonotic transmission in Cameroon. Our findings underline the existence of a potential unheeded food safety and public health threat associated with these resistant strains and reinforce the crucial importance of implementing appropriate food safety measures and promoting rational antibiotic use.

Keywords: whole genome sequencing, ESBL, Klebsiella pneumoniae, Enterobacteriaceae, food chain

\section{INTRODUCTION}

Klebsiella pneumoniae is an important Gram-negative bacillus associated with several clinical infections in humans (Perovic et al., 2014). Of particular concern is the emergence of extendedspectrum $\beta$-lactamase (ESBL) producing $K$. pneumoniae in hospital settings (Perovic et al., 2014) which has considerably increased during the last decade in response to the selection pressure of extensive antibiotic use. This resistant strain is considered a significant public health issue due to the limited therapeutic options and increased morbidity and mortality associated with it (World Health Organization [WHO], 2017). However, the concern of ESBL-producing K. pneumoniae goes beyond healthcare settings to affect various ecological niches including (food) animals, food products, soil and wastewater. In fact, humans may become colonized or infected by ESBLproducing $K$. pneumoniae upon contact with blood, saliva, feces and urine of ESBL carrier animals or consumption of contaminated water or food products (Founou et al., 2016).

The most frequent and clinically relevant ESBL genes belong to CTX-M, TEM, and SHV families, with CTX-M enzymes emerging as the predominant type. CTX-M is divided into five groups namely CTX-M-1, CTX-M-2, CTX-M-8, CTX-M-9, and CTX-M-25 according to their amino-acid identities (Perovic et al., 2014). K. pneumoniae commonly produces all three groups of enzymes but the latest public health concern has been the emergence of carbapenemase-producing $K$. pneumoniae and colistin-resistant K. pneumoniae (Hudson et al., 2014; Perovic et al., 2016). These resistance genes are generally carried on mobile genetic elements (MGEs) facilitating their dissemination within and between bacterial species (Founou et al., 2016). The presence of MGEs likely increases the proportion of serious difficult-to-treat $K$. pneumoniae infections.

The true prevalence of ESBL is not well-known in Africa and probably underestimated because of the paucity of studies in human health, animal health, and the food chain on the continent. Nevertheless, some studies, have confirmed the global distribution and the high prevalence of ESBL-producing K. pneumoniae on the continent, albeit focusing on human health sector and ignoring the animal one (Gangoue-Pieboji et al., 2005; Breurec et al., 2013; Belbel et al., 2014; Jacobson et al., 2015; Lyonga et al., 2015; Nzalie et al., 2016). For example, a multicenter study, detected ESBL-producing K. pneumoniae in public hospitals in Abidjan, Casablanca, Yaoundé, and Antananarivo with prevalence ranging from 9 to $16 \%$. The sequence type (ST) 15 and ST 11 were the most predominant K. pneumoniae clonal lineages (Breurec et al., 2013). During a national sentinel site surveillance of resistant $K$. pneumoniae, Perovic et al. (2014) reported a $68.3 \%$ prevalence of ESBLproducing K. pneumoniae in clinical samples from 2010 to 2012. Magoué et al. (2013) reported a 22.15\% prevalence of fecal ESBLproducing K. pneumoniae carriage in Ngaoundere, Cameroon, and showed the high endemicity of CTX-M-15 producers in the country. However, the genetic background of these ESBL producers were only investigated in very few of these studies. The rare African studies reporting ESBL-producing K. pneumoniae in food products emanated from Benin and Sudan where ESBLproducing $K$. pneumoniae were detected in $5.77 \%$ of vegetables (Moussé et al., 2016) and 62\% of raw milk (Badri et al., 2017), respectively.

In Cameroon, where antibiotics are used without restriction not only in the healthcare sector but also in the food production industry, the epidemiology of antibiotic resistant bacteria in food animals and associated public health implications is neglected. This study investigated the antibiotic resistance genes, virulence factors, MGEs and genetic lineages of circulating ESBL-producing K. pneumoniae strains isolated from pigs and exposed workers in Cameroonian abattoirs using whole genome sequencing (WGS), to ascertain zoonotic transmission (viz. from animals to humans and/or vice-versa) of ESBL-producing K. pneumoniae in the food chain.

\section{MATERIALS AND METHODS}

\section{Ethical Approvals}

Ethical approvals were obtained from the Biomedical Research Ethics Committee (Ref. BE365/15) and Animal Research Ethics Committee (Ref. AREC/091/015D) of the University of KwaZulu-Natal as well as from the National Ethics Committee for Research in Human Health of Cameroon (Ref. 2016/01/684/CE/CNERSH/SP) prior to the implementation of the study. Ministerial approvals were also obtained from the Cameroonian Ministry of Livestock, Fisheries and Animal Industries (Ref. 061/L/MINEPIA/SG/DREPIA/CE) and Ministry of Scientific Research and Innovation (Ref. 015/MINRESI/B00/C00/C10/C14). 


\section{Study Design and Bacterial Isolates}

From March to October 2016, a multi-center study was carried out in three slaughterhouses/markets in Cameroon and two abattoirs in South Africa, encoded for ethical reasons as SH001, SH002, SH003 and SH004 and SH005, respectively (unpublished data). Three individual samples were pooled to yield 144 nasal and 144 rectal pools representing 432 original nasal and rectal samples, respectively, collected from 432 pigs. A total of 288 swabs from the 144 nasal and 144 rectal pools constituted the final sample. Nasal and hand swabs were also collected from 82 humans in both Cameroon and South Africa. All samples were cultured on MacConkey agar supplemented with $2 \mathrm{mg} / \mathrm{L}$ cefotaxime and incubated for $18-24 \mathrm{~h}$ at $37^{\circ} \mathrm{C}$ in normal atmosphere (unpublished data). All putative ESBLproducers, were subjected to Gram staining, and the catalase and oxidase tests for phenotypic characterization of the isolates to the genus level. The isolates were thereafter phenotypically confirmed using the VITEK 2 system. The strains sequenced in this study were isolated from four pig pooled samples (PN030E4, PN089E1, PN085E1IA, and PR042E3) and three exposed workers (HH510E2I, HH517E1II, and HN523E1II) in Cameroon (unpublished data). The pig isolates, PN30E4 and PR042E3, originated from the same abattoir (SH001), although the former was collected from the nares and the latter from rectum. Likewise, the strains, PN089E1 and PN085E1IA, were both collected from the nares of pigs processed in abattoir SH002. The human strains, HH510E2I, HH517E1II, and HN523E1II, originated from three different abattoirs, SH001, SH002, and SH003, respectively, with HH510E2I and HH517E1II being collected from hands and HN523E1II from the nares. These strains were identified in a previous study, to be highly closely related via enterobacterial-repetitive-polymerase chain reaction (ERIC-PCR) analysis where they were grouped into five clusters (unpublished data). Given that we aimed to highlight and provide evidence of zoonotic transmission (i.e., from animals to humans and vice-versa) of ESBL-K. pneumoniae in the food chain, within each generated cluster, only representative isolates of intermingled strains (i.e., strains isolated from animal and/or human of the same abattoir, having high genetic relationship with those from another abattoir) were considered for WGS.

\section{Screening for ESBL}

The standard double disk synergy test (DDST), using cefotaxime and ceftazidime, alone and in combination with clavulanic acid as recommended by the Clinical Laboratory and Standards Institute (CLSI) was performed for ESBL screening. An increase in size of the inhibition zone of more than $5 \mathrm{~mm}$ in the presence of clavulanic acid was regarded as proof for ESBL production (Clinical and Laboratory Standards Institute [CLSI], 2016).

\section{Antimicrobial Susceptibility Testing}

Antimicrobial susceptibility testing was performed to determine the resistance patterns of the selected strains. Ampicillin, amoxicillin + clavulanic acid, cefuroxime, cefotaxime, ceftazidime, cefoxitin, cefepime, ertapenem, imipenem, meropenem, gentamicin, amikacin, ciprofloxacin, tigecycline, nitrofurantoin, piperacillin/tazobactam, colistin and trimethoprim-sulfamethoxazole, were tested using Vitek ${ }^{\circledR} 2$ System and Vitek ${ }^{\circledR} 2$ Gram Negative Susceptibility card (ASTN255) (BioMérieux, Marcy l'Etoile, France). The results were interpreted according to the CLSI guidelines (Clinical and Laboratory Standards Institute [CLSI], 2016) with the exception of colistin, amoxicillin + clavulanic acid, piperacillin/tazobactam, amikacin that were based on EUCAST breakpoints (The European Committee on Antimicrobial Susceptibility Testing [EUCAST], 2016) with E. coli ATCC 25922 and K. pneumoniae ATCC700603 being used as controls.

\section{DNA Isolation}

Genomic DNA (gDNA) was extracted using GenElute ${ }^{\circledR}$ bacterial genomic DNA kit (Sigma-Aldrich, St. Louis, MO, United States) according to the manufacturer's instructions. The quantification of extracted gDNA was determined on a NanoDrop spectrophotometer with verification by agarose gel electrophoresis and fluorimetric analysis $\left(\mathrm{Qubit}^{\circledR}\right)$.

\section{Genotypic Relatedness Determination of ESBL-Producing K. pneumoniae}

To establish the link of selected ESBL-producing K. pneumoniae strains from animals and humans within and between abattoirs, ERIC-PCR was performed with primers ERIC $15^{\prime}$-ATG TAA GCT CCT GGG GAT TCA C-3' ${ }^{\prime}$ and ERIC2 5'-AAG TAA GTG ACT GGG GTG AGC G-3' (Versalovic et al., 1991). Reactions were carried out in a $10 \mu \mathrm{l}$ final solution containing $0.1 \mu \mathrm{l}$ of each primer $(100 \mu \mathrm{M}), 5 \mu \mathrm{l}$ DreamTaq Green Polymerase Master Mix $2 \times$ (Thermo Fisher Scientific, South Africa), $2.8 \mu 1$ nuclease free water and $2 \mu \mathrm{l}$ DNA template and run in an Applied Biosystems 2720 programmable thermal cycler (Thermo Fisher Scientific, South Africa) with the following protocol: initial denaturation at $94^{\circ} \mathrm{C}$ for $3 \mathrm{~min}, 30$ cycles consisting of a denaturation step at $94^{\circ} \mathrm{C}$ for $30 \mathrm{~s}$, annealing at $50^{\circ} \mathrm{C}$ for $1 \mathrm{~min}$, extension at $65^{\circ} \mathrm{C}$ for $8 \mathrm{~min}$, a final extension step at $65^{\circ} \mathrm{C}$ for $16 \mathrm{~min}$ and final storage at $4^{\circ} \mathrm{C}$. ERIC profiles were digitized for analysis using Bionumerics software (version 7.6, Applied Maths, Austin, TX, United States). The similarity between each strain was determined from the homology matrix using Dice coefficient and dendrograms constructed using the algorithm Unweighted Pair-Group Method (UPGMA).

\section{Genome Sequencing}

Multiplexed paired-end libraries $(2 \times 300 \mathrm{bp})$ were prepared using the Nextera XT DNA sample preparation kit (Illumina, San Diego, CA, United States) and sequences determined on an Illumina MiSeq platform with $100 \times$ coverage at the National Institute of Communicable Diseases Sequencing Core Facility, South Africa.

\section{Genome Assembly}

The resulting raw reads were checked for quality, trimmed and de novo assembled into contigs using CLC Genomics Workbench version 10 (CLC, Bio-QIAGEN, Aarhus, Denmark) and SPAdes 
version 3.11 (Bankevich et al., 2012) to overrule any inherent shortfalls from both assemblers.

\section{Genome Analysis}

The de novo assembled reads were uploaded in GenBank and annotated using NCBI prokaryotic genome annotation pipeline and RAST 2.0 server $^{1}$ (Aziz et al., 2008) which identified encoding proteins, rRNA and tRNA, assigned functions to the genes and predicted subsystems represented in the genome. The bacterial analysis pipeline of GoSeqIt tool was also used to annotate and identify known acquired antibiotic resistant genes via ResFinder (Zankari et al., 2012), virulence factors [including capsular polysaccharide (CPS), lipopolysaccharide (LPS), adhesin, long polar fimbriae (lpfA), increased serum survival (iss), enterobactin siderophore receptor protein (iroN), biofilm, lipase, $\mathrm{ABC}$ transporter protein MchF, and gelatinase] using VirulenceFinder (Joensen et al., 2014) and MGEs through PlasmidFinder (Carattoli et al., 2014). The RAST SEED viewer was used to identify the presence of transposases and integrons flanking the $\beta$-lactamase genes (Overbeek et al., 2014). PHAge Search Tool (PHAST) server was used for the identification, annotation, and visualization of prophage sequences (Zhou et al., 2011).

\section{Multilocus Sequence Typing (MLST)}

In silico MLST-analyses was performed using the scheme of Diancourt et al. (2005), which consider allelic variation amongst seven housekeeping genes (gapa, infb, $m d h$, pgi, phoe, rpob, and tonb) to assign STs. WGS data were used to generate a K. pneumoniae MLST assignment for each isolate with new or unknown STs being sent for curation at the Klebsiella pneumoniae MLST database at the Pasteur Institute ${ }^{2}$.

\section{Whole Genome Phylogenetic Analyses}

The assembled contigs were aligned against the complete genome of $K$. pneumoniae KPN528 (CP020853) using the progressive Mauve genome alignment package version 2.3.1. Study genomes were contextualized against a collection of five relevant assembled K. pneumoniae genomes (accession numbers AYQE00000000, CP006918, CP020853, СР020847, CP020841) showing high similarity in terms of resistance genes and STs. In addition, only the complete K. pneumoniae genomes released 2015 onward were selected to ensure that the analysis depicts current evolution of ESBL-producing K. pneumoniae.

Phylogenetic analysis was based on the core genomes and performed using the Rapid large-scale prokaryote pan genome analysis (Roary) (Page et al., 2015). The maximum likelihood phylogenetic tree encompassing, country, population, sample type metadata and MLST type was generated, edited and visualized using FastTree version 2.1.7 ${ }^{3}$. In addition, the contigs were mapped against the complete genome of K. pneumoniae KPN528 (CP020853) for visualization of the genomic organization.

\footnotetext{
${ }^{1}$ http://rast.nmpdr.org

${ }^{2}$ http://bigsdb.pasteur.fr/klebsiella/klebsiella.html

${ }^{3}$ http://www.microbesonline.org/fasttree/
}

\section{Nucleotide Sequence Accession Number}

This whole-genome shotgun project PRJNA412434 of K. pneumoniae strains PN030E4, HH510E2I, HN523E1II, PN08 9E1, PR042E3, HH517E1II, and PN085E1IA has been deposited at DDBJ/EMBL/GenBank under accession numbers PDVM000 00000, PDVF00000000, PDVG00000000, PDVC00000000, PDVE 00000000, PDVU00000000, and PDVD00000000, respectively. The versions described in this paper are the versions, PDVM0 0000000.1, PDVF00000000.1, PDVG00000000.1, PDVC0000000 0.1, PDVE00000000.1, PDVU00000000.1, and PDVD00000000.1, respectively.

\section{RESULTS}

\section{Phenotypic Analyses}

Out of the 144 pooled nasal samples (three nasal swabs each) and 144 pooled rectal samples (three rectal swabs each) taken from 432 pigs in Cameroon and South Africa, ESBL-producing Enterobacteriaceae were detected in 108/144 (75\%) and 102/144 (71\%) nasal and rectal pools, respectively (unpublished data). Carriage of ESBL-producing Enterobacteriaceae was observed in Cameroonian workers only. Multiple colonies (up to 10) were isolated from both populations and countries, but due to financial constraints, post-stratification allowed the selection of 158 ESBL-PE in pigs and 71 in exposed workers, for phenotypic analysis (unpublished data). Out of these, ESBLproducing $K$. pneumoniae were only detected in Cameroon with $21.52 \%(34 / 158)$ and $11.26 \%(8 / 71)$ detected in pigs and exposed workers, respectively (unpublished data). None of the South African pig or human samples were positive for ESBL-producing $K$. pneumoniae. Table 1 summarizes relevant population data, specimen source, phenotypic, and genotypic characteristics for these isolates. All isolates displayed reduced susceptibility to the amino-penicillins, cephalosporins, trimethoprim-sulfamethoxazole, with various resistance to gentamicin $(n=5 ; 71 \%)$, ciprofloxacin $(n=1 ; 14 \%)$ and nitrofurantoin $(n=1 ; 14 \%)$. The antimicrobial susceptibility results of the ESBL-producing $K$. pneumoniae isolates are summarized in Table 2, with the resistance observed being corroborated with WGS analyses.

\section{Genotypic Analyses}

All isolates carried sulfonamide (sul1), fosfomycin (fosA) and quinolone (oqxA and $o q x \mathrm{~B})$ resistance genes. Various $\beta$-lactamase encoding determinants were detected with bla $_{\mathrm{CTX}-\mathrm{M}-15}(86 \%)$, bla $a_{\mathrm{TEM}-1 \mathrm{~B}}(57 \%)$ and bla SCO-1 $(43 \%)$ being the most prevalent. Four (PN089E1, PN085E1IA, HH517E1II, and HH510E2II) and three (PR042E3, PN030E4, and HN523E1II) isolates concomitantly harbored four and three $\beta$-lactamase encoding genes. Likewise, six (86\%) strains harbored $d f \mathrm{~A} 15$ gene responsible for trimethoprim resistance, while co-presence of $s t r \mathrm{~A}$ and $s t r \mathrm{~B}$ encoding for aminoglycoside resistance was observed in five (71\%) isolates as was the tet(A) gene responsible for tetracycline resistance. Interestingly, bla $a_{\mathrm{OXA}-9}, b l a_{\mathrm{LEN} 12}, b l a_{\mathrm{SHV}-134}, b l a_{\mathrm{TEM}-1 \mathrm{~A}}$ were observed in the unique $K$. pneumoniae ST307 strain as were $a a c\left(6^{\prime}\right) I b-c r$, 
catA2, sul2, tet(D), $\operatorname{drf} \mathrm{A} 27$ and $A R R-3$ encoding resistance to aminoglycosides, phenicols, sulfonamides, tetracycline, trimethoprim and rifampicin, respectively. Similarly, the $\beta$-lactamase genes $b l a_{\mathrm{TEM}-116}$ and $b l_{\mathrm{SHV}-28}$, and plasmid mediated quinolone resistance (PMQR) genes QnrB1 were only identified in the two $K$. pneumoniae ST14 strains. None of the $K$. pneumoniae isolates harbored virulence genes.

\section{Multilocus Sequence Typing}

In silico MLST-analyses revealed that $K$. pneumoniae strains belonging to five different STs, namely ST14 $(n=2)$, ST39 $(n=2)$, a single-locus variant ST307 and two new sequences. The two K. pneumoniae ST14 strains were isolated from a pig pooled nasal sample (PN30E4) and a human nasal swab (HN523E1II) located in two different abattoirs, SH001 and SH003, respectively (Table 1). The K. pneumoniae ST39 strains, HH517E1II, and PN085E1IA, were also detected from an exposed worker and pooled nasal samples but both from the same abattoir SH002. In our collection of seven ESBL-producing $K$. pneumoniae isolates, two strains, PR042E3 and PN089E1 isolated from pigs in two different abattoirs (SH001 and SH002), had a novel combination of known K. pneumoniae MLST alleles, that were assigned as ST2958 $(n=1)$ and ST2959 $(n=1)$, respectively. The sole K. pneumoniae ST307 strain was isolated from hand of an exposed worker and harbored a total of 22 resistance determinants encoding resistance to nine antibiotic classes.

\section{Phylogenetic Analysis}

The phylogenetic analysis confirmed the intermingled reservoir of ESBL-producing K. pneumoniae strains and revealed that our strains fall within two clades of international $K$. pneumoniae isolates. Figure $\mathbf{1}$ demonstrates considerable similarity between our collection of K. pneumoniae ST14 and three strains CP020841 (ST37), CP006918 (ST258), and CP020847 (ST906), all isolated from clinically ill humans in United States. Similarly, the K. pneumoniae ST39 strains isolated from nares of healthy pig (PN085E1IA) and the hand of healthy human (HH517E1II) in abattoir SH002, were closely related K. pneumoniae ST307 (HH510E2I) isolated from hand of a human in SH001 and a K. pneumoniae ST336 isolated in clinically ill patient in Lebanon (Figure 1). Figure 2 shows the genomic organization of the ESBL-producing K. pneumoniae HN523E1II mapped against the complete genome of K. pneumoniae KPN528 (CP020853).

\section{Detection of Plasmids and Phage-Associated Regions}

PlasmidFinder revealed that the colRNAI plasmid replicon type was identified in all strains whereas the $\operatorname{IncFIB}(\mathrm{K})$ plasmid incompatibility group was detected in six $(86 \%)$ isolates. The two K. pneumoniae ST14 strains, PN030E4 and HN523E1II, concomitantly harbored two colRNAI plasmid replicon types as well as two IncFIA(HI1) and IncFIB(K) plasmid incompatibility groups, with the strain PN030E4 additionally harboring the IncY plasmid. Likewise, the K. pneumoniae ST307 harbored two colRNAI replicons as well as FIA (pBK30683) and IncR plasmid incompatibility groups while the $K$. pneumoniae ST 39 carried four colRNAI replicons along with IncHI1B and IncFIB $(\mathrm{K})$ plasmid incompatibility groups. In silico plasmid MLST-analyses assigned the IncF plasmid incompatibility group as belonging to various STs including [K1:A13-like:B-], [K-:A10-like:B-], [K-:A13:B-] while the IncH plasmid belonged to a non-typeable ST.

With regard to the phage-associated regions, all strains hosted at least one intact bacteriophage (Table 3). Shigel SfII, pseudo JBD44, Entero lato and Escher HK639 were the predominant intact bacteriophages. Six phage regions were identified in one K. pneumoniae ST14 (HN523E1II) isolated from hand of a worker using PHAST algorithm, while the other ST14 (PN030E4) and one ST39 (PN085E1IA) observed in pigs hosted three phage

TABLE 1 | Summary of population, sample type, phenotypic and genotypic characteristics of ESBL-producing $K$. pneumoniae isolates.

\begin{tabular}{|c|c|c|c|c|c|c|}
\hline Isolate name & Host & Sample type & Abattoir & Antibiotic resistance genes & Plasmids & MLST \\
\hline PN030E4 & Pig & Nasal swab & SH001 & $\begin{array}{l}\text { strA, strB, bla } \text { TEM-116, blas }_{\mathrm{SHV}-28}, \text { bla } \mathrm{CTX}-\mathrm{M}-15, \text { oqxA, } \\
\text { oqxB, QnrB1, fosA, sul1, sul2, tet(A), dfrA15 }\end{array}$ & 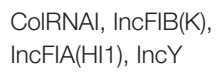 & ST14 \\
\hline HN523E1II & Human & Nasal swab & $\mathrm{SHOO3}$ & $\begin{array}{l}\text { strA, strB, bla } \mathrm{TEM}-116, \text { bla } \mathrm{SHV}-28, \text { bla } \mathrm{CTX}-\mathrm{M}-15, \text { oqxA, } \\
\text { oqxB, QnrB1, fosA, sul1, dfrA15 }\end{array}$ & $\begin{array}{l}\text { ColRNAI, IncFIB }(K) \\
\operatorname{IncFIA}(\mathrm{HI} 1)\end{array}$ & ST14 \\
\hline HH517E1II & Human & Hand swab & $\mathrm{SHOO2}$ & $\begin{array}{l}\text { aac(3)-lla, aadA1, bla } \mathrm{TEM}_{\mathrm{TE}} \text {, blas } \mathrm{a}_{\mathrm{SV}-11}, \text { bla } \mathrm{CTX}-\mathrm{M}-15 \\
\text { bla }_{\mathrm{SCO}-1}, \text { oqxA, oqxB, fosA, sul1, tet(A), dfrA15 }\end{array}$ & $\begin{array}{l}\text { ColRNAl, IncFIB }(K) \\
\text { IncHI1B }\end{array}$ & ST39 \\
\hline PN085E1IA & Pig & Nasal swab & $\mathrm{SHOO2}$ & $\begin{array}{l}\text { aac(3)-lla, aadA1, strA, strB, bla }{ }_{\mathrm{TEM}-1 \mathrm{~B}} \text {, bla } \mathrm{SHV}-27, \\
\text { bla }_{\mathrm{ScO}-1} \text {, bla } \mathrm{CTX}-\mathrm{M}-15, \text { oqxA, oqxB, fosA, catA1, sul1, } \\
\text { sul2, tet(A), dfrA15 }\end{array}$ & $\begin{array}{l}\text { ColRNAI, IncFIB }(K) \\
\text { IncFII(K), } \\
\text { FIA(pBK30683) }\end{array}$ & ST39 \\
\hline PR042E3 & Pig & Rectal swab & SH001 & $\begin{array}{l}\text { aac(3)-lla, aadA1, strA, strB, bla }{ }_{\mathrm{TEM}-1 \mathrm{~B}}, \text { blas } \mathrm{SHV}-1 \\
\text { bla }\end{array}$ & ColRNAI, IncFIB(K) & ST2958 \\
\hline PN089E1 & Pig & Nasal swab & SH0O2 & 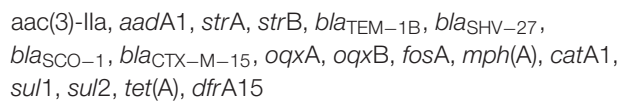 & $\begin{array}{l}\text { ColRNAl, IncFIB(K), } \\
\text { IncR, ColE10 }\end{array}$ & ST2959 \\
\hline HH510E2I & Human & Hand swab & SH001 & 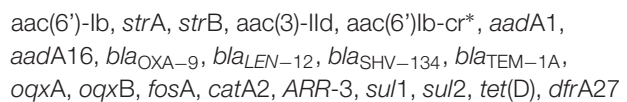 & $\begin{array}{l}\text { ColRNAI, IncR, } \\
\text { FIA(pBK30683) }\end{array}$ & ST307 \\
\hline
\end{tabular}

*aac(6')lb-cr: confers concomitant resistance to aminoglycosides and fluoroquinolones. 


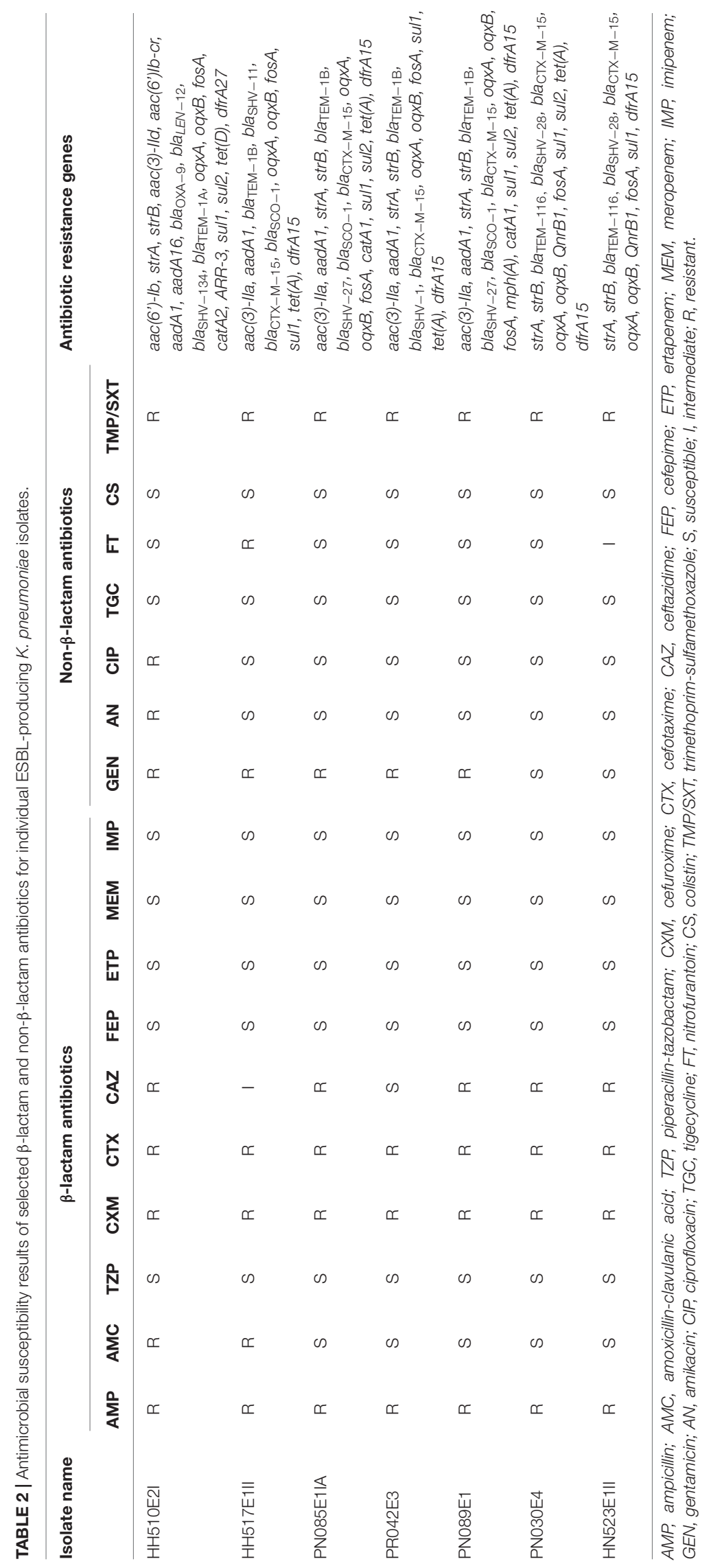


TABLE 3 | Distribution of intact prophage regions among the ESBL-producing $K$. pneumoniae strains.

\begin{tabular}{|c|c|c|c|c|c|}
\hline Isolate name & Region ${ }^{a}$ & Length $^{b}$ (kb) & No CDS & GC $\%$ & Phage (hit genes count) ${ }^{c}$ \\
\hline HH510E2I & 1 & 37.6 & 47 & 51.42 & Salmon 103203 sal5 (13) \\
\hline HH517E1II & 1 & 39.3 & 52 & 52.13 & Salmon 64795 sal3 (15) \\
\hline \multirow[t]{6}{*}{ HN523E1II } & 1 & 30.3 & 37 & 51.02 & Entero mEp390 (6) \\
\hline & 2 & 17.1 & 20 & 48.37 & Entero lato (7) \\
\hline & 3 & 27.6 & 12 & 53.30 & Shigel Sfll (8) \\
\hline & 4 & 17.4 & 26 & 54.84 & Shigel Sfll (18) \\
\hline & 5 & 58.6 & 44 & 53.75 & Escher HK639 (18) \\
\hline & 6 & 29.8 & 35 & 51.86 & Entero c 1 (9) \\
\hline \multirow[t]{3}{*}{ PN030E4 } & 1 & 29.6 & 35 & 51.54 & Brucel BiPBO1 (6) \\
\hline & 2 & 23 & 28 & 53.03 & Shigel Sfll (20) \\
\hline & 3 & 48.6 & 49 & 53.27 & Escher HK639 (19) \\
\hline \multirow[t]{3}{*}{ PN085E1IA } & 1 & 27.4 & 28 & 52.12 & Pseudo JBD44 (9) \\
\hline & 2 & 31.4 & 25 & 52.93 & Entero c 1 (12) \\
\hline & 3 & 33.1 & 12 & 49.24 & Entero lato (7) \\
\hline \multirow[t]{2}{*}{ PR089E1 } & 1 & 47.6 & 41 & 50.80 & Salmon 118970 sal3 (11) \\
\hline & 2 & 27 & 34 & 55.25 & Salmon Fels 2 (22) \\
\hline PR042E3 & 1 & 53.1 & 57 & 52.17 & Pseudo JBD44 (13) \\
\hline
\end{tabular}

a Intact prophage region; ${ }^{b}$ Region length of intact prophage; ${ }^{c}$ Phage with the highest number of CDS in the region and the number of gene counts in brackets.

regions each. Figure 3 shows the genomic structure of the phage Escher HK639 in the K. pneumoniae ST14 (HN523E1II).

\section{DISCUSSION}

In this study, antibiotic resistance genes, virulence factors, MGEs and genetic lineages of seven circulating and closely related ESBL-producing K. pneumoniae strains isolated from pigs and occupationally exposed workers in Cameroonian abattoirs were investigated using WGS.

ESBL-producing K. pneumoniae have been observed at diverse rates in clinical samples in the Ivory Coast (16\%), Morocco (13\%), Cameroon (10\%) and Madagascar (9\%) (Breurec et al., 2013). They were also responsible of community-acquired urinary tract infections in a Cameroonian city with an incidence of $16.4 \%$ (Nzalie et al., 2016). Our study, similarly revealed ESBL-producing K. pneumoniae strains in pigs $(27.64 \%)$ and exposed workers (16.66\%) in Cameroon as the first report ESBL-producing $K$. pneumoniae isolates in food animals and occupationally exposed workers in the country. The ESBLproducing $K$. pneumoniae strains were mainly circulating in two clonal lineages since four out of seven isolated strains belonged to the ST14 $(n=2)$ and ST39 $(n=2)$. To the best of our knowledge, this is the first evidence of a porcine ESBLproducing $K$. pneumoniae reservoir in this country. Moreover, the first reports of $b l a_{\mathrm{CTX}-\mathrm{M}-15}, b l a_{\mathrm{TEM}-1 \mathrm{~B}}, b l a_{\mathrm{SHV}-11}, b l a_{\mathrm{SCO}-1}$ producing $K$. pneumoniae ST39 and bla $a_{\mathrm{CTX}-\mathrm{M}-15}, b l a_{\mathrm{TEM}-116}$, bla $a_{\mathrm{SHV}-28}$ producing K. pneumoniae ST14 in pigs and humans in Cameroon are presented here.

The K. pneumoniae ST14 isolates were found to be resistant to ampicillin, cefuroxime, cefuroxime-axetil, cefotaxime, ceftazidime and trimethoprim-sulfamethoxazole. This resistance phenotype was corroborated by the identification of the CTX-M15, SHV-28, and TEM-116 genes by WGS which also elucidated with multiple resistant determinants to non- $\beta$-lactam antibiotics, notably the aminoglycoside resistant genes (strA, strB), plasmidmediated quinolone resistance genes (QnrB1, oq $x \mathrm{~A}$, oq $x \mathrm{~B})$, fosfomycin resistant gene (fos $\mathrm{A}$ ), and sulfonamide resistant gene (sul1 and sul2) which were not phenotypically evident. Although the two K. pneumoniae ST39 isolates displayed similar phenotypic profiles, these were attributed to different resistance gene permutations. For example, the pig strain PN085E1IA harbored genes encoding for aminoglycoside [aad1, aac(3)-IIa], fluoroquinolones (oqxA, oq $x \mathrm{~B})$, fosfomycin (fos $\mathrm{A})$, tetracycline [tet(A)], trimethoprim (dfrA15) and sulfonamide (sul1, sul2) resistance alongside blaCTX-M-15, bla $a_{\mathrm{TEM}-1 \mathrm{~B}}$ and bla $a_{\mathrm{SHV}-1}$ while aad1, aac(3)-IIa, oqxA, oqxB, fosA, sul1, tet(A), dfrA15, bla $a_{\mathrm{TEM}-1 \mathrm{~B}}, b l a_{\mathrm{CTX}-\mathrm{M}-15}, b l a_{\mathrm{SCO}-1}, b l a_{\mathrm{SHV}-11}$ were observed in the human strain HH517E1II. CTX-M-15 detection is consistent with a multicenter study conducted in five African (including Yaoundé) and two Vietnamese towns where it was detected in $74 \%$ of isolates and was the predominant ESBL among the African isolates (Breurec et al., 2013). This study further reported the predominance of $\mathrm{Q} n \mathrm{rB}$ determinant among the African strains (Breurec et al., 2013). In addition, CTX-M-15-producing $K$. pneumoniae hosted on a plasmid has already been reported in patients with clinical urinary tract infections in Cameroon (Gangoue-Pieboji et al., 2005), confirming the widespread dissemination of this ESBL type. The preponderance of CTXM-15 as the main ESBL genes $(85.71 \%)$ in our $K$. pneumoniae isolates confirms that CTX-M-15 is currently the most largely distributed CTX-M enzyme worldwide. The potential role of $K$. pneumoniae as a reservoir for $\beta$-lactam and non- $\beta$-lactam resistance determinants is a major concern in countries with inadequate antibiotic resistance (ABR) surveillance, prevention 


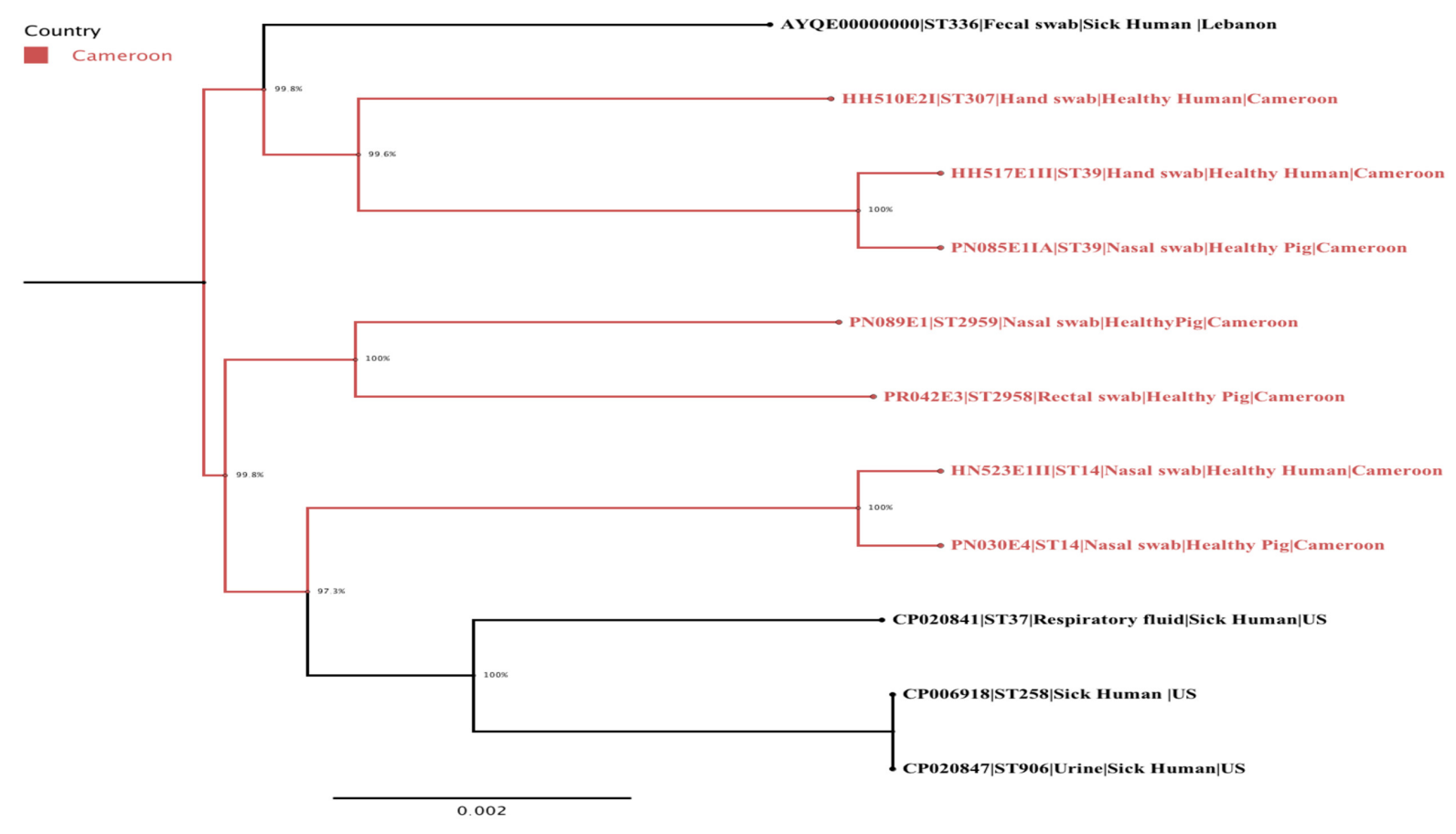

FIGURE 1 | A maximum-likelihood phylogenetic tree of ESBL-producing $K$. pneumoniae isolates generated using FastTree version 2.1.7. The Cameroonian isolates are colored in red. The following information is also provided for each isolate: name/reference, sequence type, type of sample, population, and country.

and containment measures such as Cameroon. Key factors favoring the emergence and spread of ABR in the food chain in the country include irrational antibiotic use on farms, poor sanitary and feeding practices, sub-optimal transport conditions, lack of veterinarian control, inadequate diagnostic facilities, substandard quality of antibiotics, lack of antimicrobial monitoring and poor biosecurity measures (Ndebi et al., 2009).

Our study showed that both ST14 and ST39 demonstrated overlap and intermingled populations between pig- and humansources within and across abattoirs (Figure 1). Specifically, the K. pneumoniae ST14 strains colonized nares of both human and pigs located in two different abattoirs (SH001 and SH003) whereas $K$. pneumoniae ST39 was detected in nares of pigs and the hand of a worker present in the same abattoir, SH002 (Figure 1). This could be associated with neglected hygienic practices prevailing during production, transport, storage and/or retail stages. The virtual absence of physical barriers between community and healthcare settings in this country along with poverty and limited education may also be important contributory factors.

The ST14 and ST39 clonal lineages are a major cause of nosocomial infections and outbreak situations around the world, although their evolutionary emergence is somewhat poorly documented in developing countries. In fact, OXA-181producing K. pneumoniae ST14 was detected in South Africa where it was responsible of an outbreak of among hospitalized patients in a tertiary hospital (Jacobson et al., 2015), whereas, a multidrug resistant and biofilm producing $K$. pneumoniae strain belonging to the ST14 was detected in India also at tertiary care (Rafiq et al., 2016). Similarly, the K. pneumoniae ST39 was responsible for an outbreak in a pediatric hospital in Algeria (Belbel et al., 2014). The isolation of these STs always urged the implementation of stringent infection and control measures and ongoing surveillance of antibiotic resistance in hospital settings. Similar strict interventions should thus be undertaken in the food production industry if we are to successfully contain their clonal dissemination in the food chain.

The detection of the international K. pneumoniae ST307 in a human isolate is further evidence of the wide and increasing spread of ESBL-producing bacteria in the country. The K. pneumoniae ST307 has been recognized as candidate for becoming one of the prevalent high-risk and clinically relevant clones since its worldwide emergence during the last five years (Villa et al., 2017). The K. pneumoniae ST307 lineage is generally capsulated, displays higher resistance to complementmediating killing, has novel virulence arrays and is associated with CTX-M-15 and KPC encoding plasmids (Villa et al., 2017). Accordingly, Klebsiella pneumoniae Carbapenemase (KPC)producing $K$. pneumoniae ST307 carrying a self-transferable plasmid (IncX3-type) was detected among clinical specimens during a nosocomial outbreak in South Korea (Kim et al., 2017) as was KPC-producing K. pneumoniae ST307 harboring pKPN307 plasmid (Villa et al., 2017). In contrast to these reports, the K. pneumoniae ST307 detected in our study did not harbor the CTX-M-15 and KPC enzymes nor the IncX plasmid but rather TEM-1A, LEN12, OXA- 9 and SHV-134 as $\beta$-lactamases, and colRNAI, IncR and FIA (pBK30683) plasmid incompatibility groups, suggesting a different phylogenetic evolution. The ability 


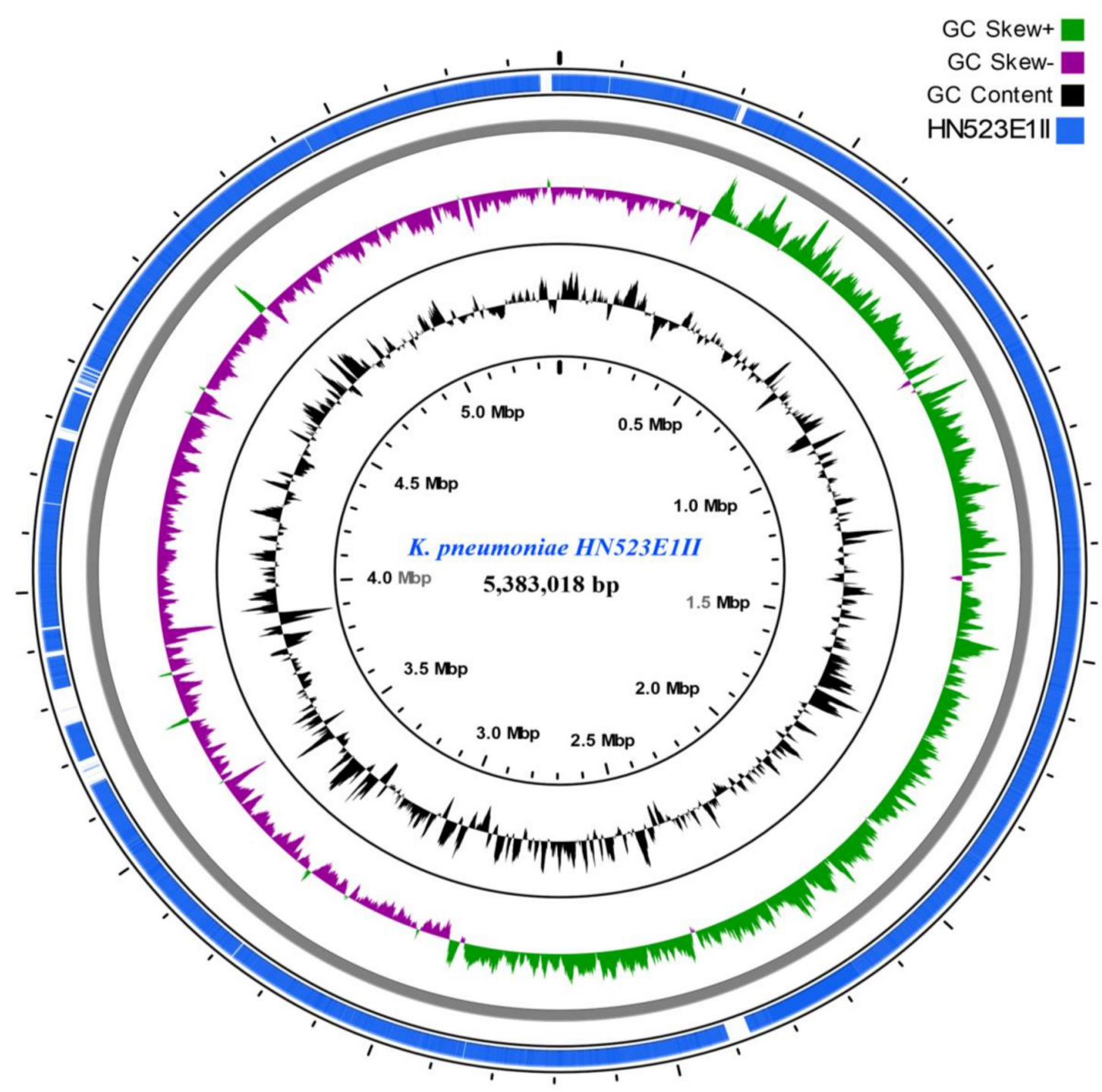

FIGURE 2 | ESBL-producing K. pneumoniae HN523E1|l ring representation using CGView Server V 1.0 (Grant et al., 2012 ). The inner ring displays the percent of identity comparing K. pneumoniae HN523E1II and the complete genome of K. pneumoniae KPN528 (CP020853). The two next (inner) rings display the GC content and GC skew, respectively. The last outer ring indicates the genome K. pneumoniae HN523E1II.

of this clonal lineage to acquire novel genetic features may contribute to its increased persistence in the environment and highlights its potential public health threat.

Only K. pneumoniae ST11 and ST15 were detected from clinical samples in Yaoundé, Cameroon to date. The detection of two new genome sequences K. pneumoniae ST2958 and ST2959 in pigs with different antibiotic resistance profiles and genes, improve our understanding and scope of the molecular epidemiology and evolution of resistant bacteria in the country. The emergence of these ESBL-producing $K$. pneumoniae in pigs and exposed workers within and between abattoirs in Cameroon is of great significance as it confirms their active clonal dissemination via direct contact, and suggests their indirect spread throughout the food chain in the country. These findings further suggest that pigs, pork, and abattoir workers represent a potential reservoir and source of foodborne ESBL-producing $K$. pneumoniae infections in Cameroon and reinforce the crucial importance of implementing appropriate food safety measures and promoting rational antibiotic use.

All isolates except HH510E2I (MIC $\geq 4 \mu \mathrm{g} / \mathrm{ml}$ ) were susceptible to ciprofloxacin ( $\mathrm{MIC} \leq 0.5 \mu \mathrm{g} / \mathrm{ml}$ ) suggesting that they might not contain gene encoding for resistance to fluoroquinolones, but oqxA and oqxB were detected in all isolates. Similarly, although not expressing phenotypic resistance to gentamicin ( $\mathrm{MIC} \leq 1 \mu \mathrm{g} / \mathrm{ml})$, amikacin $(\mathrm{MIC} \leq 2 \mu \mathrm{g} / \mathrm{ml})$ and ciprofloxacin (MIC $\leq 0.25 \mu \mathrm{g} / \mathrm{ml}$ ), the pig and human isolates, PN030E4 and HN523E1II, harbored strA and strB encoding for resistance to aminoglycoside together with the fluoroquinolone 


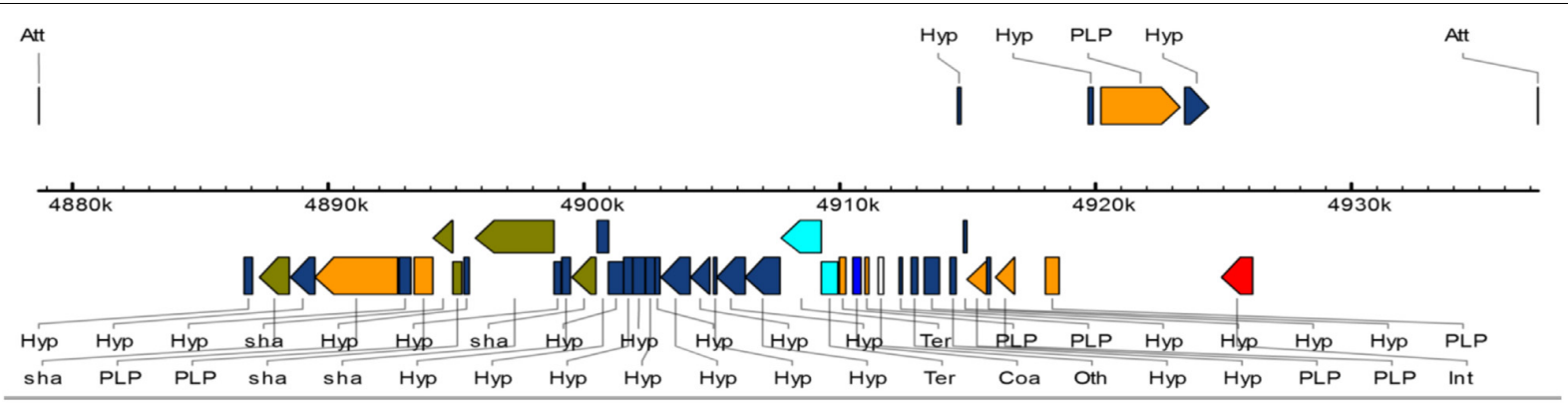

Identified CDS types:
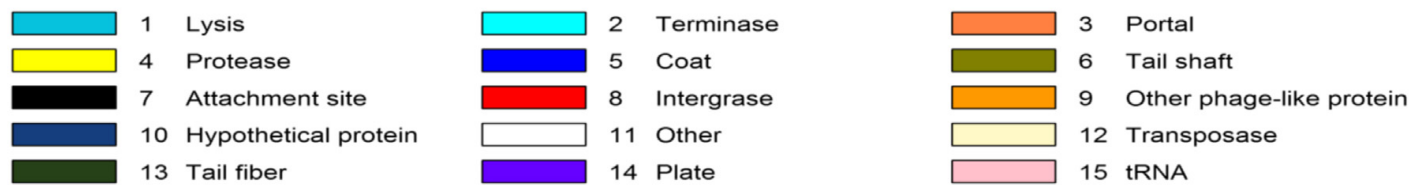

FIGURE 3 | Linear view of the prophage Escher HK639 isolated from the K. pneumoniae ST14 (HN523E1II). Putative genes are colored according to the predicted functions of their products.

resistance genes $o q x \mathrm{~A}, o q x \mathrm{~B}$ and $\mathrm{Q} n r \mathrm{~B} 1$. This finding thus indicate that these genes might be "silent" in ESBL-producing K. pneumoniae circulating in pigs and humans in Cameroon, and could likely be triggered in response to the selective pressure of widespread antibiotic use.

FosA gene a glutathione $S$-transferase that causes enzymatic inactivation of and resistance to fosfomycin, was detected in six out of seven isolates in our study with 100\% homology. Fosfomycin is a broad-spectrum antibiotic used extensively in Europe and Africa for treatment of uncomplicated urinary tract infections (Xu et al., 2011). It is receiving renewed interest globally as a therapeutic option for the treatment of infections caused by carbapenem-resistant Enterobacteriaceae. Our finding of chromosomal fosA gene in $85.71 \%$ our isolates concurs with a report which revealed that several Gram-negative species including K. pneumoniae, K. oxytoca, Enterobacter cloacae, Enterobacter aerogenes, Serratia marcescens, carry fosA gene on their chromosome and are species with intrinsic reduced susceptibility or resistance to fosfomycin (Ito et al., 2017). The widely distribution of fos A gene in our study further suggests that $K$. pneumoniae could serve as a reservoir for this gene and facilitate its dissemination to species lacking fos A such as E. coli in the country. Although the contextualization of this finding is difficult due to the scarcity of molecular epidemiological studies in Africa, this finding concurs with several studies from Asian (Wachino et al., 2010; Hou et al., 2013; Chan et al., 2014) and European countries (Benzerara et al., 2017) which showed the presence of multiple lineages of fosA producing ESBL-E. coli. Any interest in repurposing use of old antibiotics should thus be considered with caution and in light of existing environmental reservoirs of resistance genes.

The CGview analyses showed that major parts of the K. pneumoniae ST14 (HN523E1II), are present within the reference genome KPN528 (CP020853) (Figure 2). However, some discrepancies in both identity and coverage were observed, as part of the rings are lighter colored or missing, implicating the presence of other genetic elements or independent evolution of this isolate. This suggests that closure of our genomes would be essential to decipher and understand the evolutionary biology of ESBL-producing K. pneumoniae. Besides, investigation of the resistance genes and comparison along with the phage-associated regions harbored by our isolates, did not reveal the presence of a prophage-encoding resistance genes. This suggests that prophages are unlikely to act as vectors for the dissemination of resistance within our isolates. Nonetheless, the acquisition of MGEs including the plasmids and bacteriophages contribute to the phenotypic and genetic plasticity of their bacterial host and can act as vector for the transfer of resistance determinants and virulence factors leading to increased prevalence. Routine screening for ESBL-producing $K$. pneumoniae colonization in food animals, exposed workers, farms, abattoirs and food products is thus essential for its effective containment.

Even though our findings may not be extrapolated to the overall situation of the country, it is noteworthy that these highlight a serious food safety threat as the study took place in the main abattoirs of the country's capital. Further highresolution genotyping studies of ESBL-producing K. pneumoniae collected over larger temporal and spatial scales are required to better understand the evolution, molecular epidemiology, and transmission dynamics of these resistant isolates.

\section{CONCLUSION}

Our study shows that ESBL-producing $K$. pneumoniae is actively disseminating in pigs and occupationally exposed workers 
in Cameroonian abattoirs and is probably under-estimated considering the absence of molecular epidemiological studies. It underlines the existence of a potential unheeded food safety and public health threat associated with these resistant strains in the country especially if they spread to susceptible people such as immunocompromised. Ongoing efforts and further well-designed epidemiological studies to understand the epidemiology, transmission dynamics and pathways, risk factors and public health implications associated with the food animal reservoir of ESBL-producing bacteria are essential to inform effective interventions for their containment.

\section{AUTHOR CONTRIBUTIONS}

LF co-conceptualized the study, undertook sample collection, microbiological laboratory and data analyses, prepared tables and figures, interpreted results, contributed to bioinformatics analysis, and drafted the manuscript. RF undertook sample collection, microbiological laboratory analyses, contributed bioinformatics analysis and vetting of the results. MA undertook bioinformatics analyses. AI performed WGS analysis. CD took part in the design of the study, provided material, equipment and reagents, undertook critical revision of the manuscript and coordinated the field implementation. SE co-conceptualized the

\section{REFERENCES}

Aziz, R. K., Bartels, D., Best, A. A., DeJongh, M., Disz, T., Edwards, R. A., et al. (2008). The RAST server: rapid annotations using subsystems technology. BMC Genomics 9:75. doi: 10.1186/1471-2164-9-75

Badri, A. M., Ibrahim, I. T., Mohamed, S. G., Garbi, M. I., Kabbashi, A. S., and Arbab, M. H. (2017). Prevalence of extended spectrum beta lactamase (ESBL) producing Escherichia coli, and Klebsiella pneumoniae isolated from raw milk samples in Al Jazirah state, Sudan. Mol. Biol. 7:1000201. doi: 10.4172/21689547.1000201

Bankevich, A., Nurk, S., Antipov, D., Gurevich, A. A., Dvorkin, M., Kulikov, A. S., et al. (2012). SPAdes: a new genome assembly algorithm and its applications to single-cell sequencing. J. Comput. Biol. 19, 455-477. doi: 10.1089/cmb.2012. 0021

Belbel, Z., Chettibi, H., Dekhil, M., Ladjama, A., Nedjai, S., and Rolain, J.-M. (2014). Outbreak of an armA methyltransferase-producing ST39 Klebsiella pneumoniae clone in a pediatric Algerian Hospital. Microb. Drug Resist. 20, 310-315. doi: $10.1089 / \mathrm{mdr} .2013 .0193$

Benzerara, Y., Gallah, S., Hommeril, B., Genel, N., Decré, D., Rottman, M., et al. (2017). Emergence of plasmid-mediated fosfomycin-resistance genes among Escherichia coli isolates, France. Emerg. Infect. Dis. 23, 1564-1567. doi: 10.3201/ eid2309.170560

Breurec, S., Guessennd, N., Timinouni, M., Le, T. A., Cao, V., Ngandjio, A., et al. (2013). Klebsiella pneumoniae resistant to third-generation cephalosporins in five African and two Vietnamese major towns: multiclonal population structure with two major international clonal groups, CG15 and CG258. Clin. Microbiol. Infect. 19, 349-355. doi: 10.1111/j.1469-0691.2012. 03805.x

Carattoli, A., Zankari, E., García-Fernández, A., Voldby, L., Lund, O., Villa, L., et al. (2014). In silico detection and typing of plasmids using PlasmidFinder and plasmid multilocus sequence typing. Antimicrob. Agents Chemother. 58, 3895-3903. doi: 10.1128/AAC.02412-14

Chan, J., Lo, W. U., Chow, K. H., Lai, E. L., Law, P. Y., and Ho, P. L. (2014). Clonal diversity of Escherichia coli isolates carrying plasmid-mediated fosfomycin resistance gene fosA3 from livestock and other animals. Antimicrob. Agents Chemother. 58, 5638-5639. doi: 10.1128/AAC.02700-14 study and undertook critical revision of the manuscript. All authors read and approved the final manuscript.

\section{FUNDING}

LF and RF are funded by the Antimicrobial Research Unit (ARU) and College of Health Sciences (CHS) of the University of KwaZulu-Natal, South Africa. The National Research Foundation funded this study through the NRF Incentive Funding for Rated Researchers (Grant No. 85595), the NRF Competitive Grant for Rated Researchers (Grant No. 106063) and the DST/NRF South African Research Chair in Antibiotic Resistance and One Health (Grant No. 98342) awarded to SE. The South African Medical Research Council also funded the study through the Self-Initiated Researcher (SIR) Grant awarded to SE.

\section{ACKNOWLEDGMENTS}

We are grateful to the NCBI GENBANK submission staff for help with genome upload, decontamination and deposition procedures. We thank the team of curators of the Institute Pasteur MLST system (Paris, France) for importing novel alleles, profiles and/or isolates at http://bigsdb.web.pasteur.fr.

Clinical and Laboratory Standards Institute [CLSI] (2016). Performance Standards for Antimicrobial Susceptibility Testing; Twenty-Six Informational Supplement. CLSI Document M100-S26. Wayne, PA: Clinical and Laboratory Standards Institute.

Diancourt, L., Passet, V., Verhoef, J., Grimont, P. A., and Brisse, S. (2005). Multilocus sequence typing of Klebsiella pneumoniae nosocomial isolates. J. Clin. Microbiol. 43, 4178-4182. doi: 10.1128/jcm.43.8.4178-4182. 2005

Founou, L. L., Founou, R. C., and Essack, S. Y. (2016). Antibiotic resistance in the food chain: a developing country-perspective. Front. Microbiol. 7:1881. doi: 10.3389/fmicb.2016.01881

Gangoue-Pieboji, J., Miriagou, V., Vourli, S., Tzelepi, E., Ngassam, P., and Tzouvelekis, L. S. (2005). Emergence of CTX-M-15-producing enterobacteria in cameroon and characterization of a bla (CTX-M-15)-carrying element. Antimicrob. Agents Chemother. 49, 441-443. doi: 10.1128/AAC.49.1.441-443. 2005

Grant, J. R., Arantes, A. S., and Stothard, P. (2012). Comparing thousands of circular genomes using the CGView comparison tool. BMC Genomics 13:202. doi: 10.1186/1471-2164-13-202

Hou, J., Yang, X., Zeng, Z., Lv, L., Yang, T., Lin, D., et al. (2013). Detection of the plasmid-encoded fosfomycin resistance gene fosA3 in Escherichia coli of food-animal origin. J. Antimicrob. Chemother. 68, 766-770. doi: 10.1093/jac/ dks465

Hudson, C., Bent, Z., Meagher, R., and Williams, K. (2014). Resistance determinants and mobile genetic elements of an NDM-1-encoding Klebsiella pneumoniae strain. PLOS ONE 9:e99209. doi: 10.1371/journal.pone.00 99209

Ito, R., Mustapha, M. M., Tomich, A. D., Callaghan, J. D., McElheny, C. L., Mettus, R. T., et al. (2017). Widespread fosfomycin resistance in gram-negative bacteria attributable to the chromosomal fosA gene. mBio 8:e00749-17. doi: 10.1128/ mBio.00749-17

Jacobson, R. K., Manesen, M. R., Moodley, C., Smith, M., Williams, S. G., Nicol, M. P., et al. (2015). Molecular characterisation and epidemiological investigation of an outbreak of blaOXA-181 carbapenemase-producing isolates of Klebsiella pneumoniae in South Africa. S. Afr. Med. J. 105, 1030-1035. doi: 10.7196/SAMJ.2015.v105i12.9926 
Joensen, K. G., Scheutz, F., Lund, O., Hasman, H., Kaas, R. S., Nielsen, E. M., et al. (2014). Real-time whole-genome sequencing for routine typing, surveillance, and outbreak detection of verotoxigenic Escherichia coli. J. Clin. Microbiol. 52, 1501-1510. doi: 10.1128/JCM.03617-13

Kim, J. O., Song, S. A., Yoon, E. J., Shin, J. H., Lee, H., Jeong, S. H., et al. (2017). Outbreak of KPC-2-producing Enterobacteriaceae caused by clonal dissemination of Klebsiella pneumoniae ST307 carrying an IncX3-type plasmid harboring a truncated Tn4401a. Diagn. Microbiol. Infect. Dis. 87, 343-348. doi: 10.1016/j.diagmicrobio.2016.12.012

Lyonga, E. E., Toukam, M., Nkenfou, C., Gonsu, H. K., Assoumou, M.-C. O., Mesembe, M., et al. (2015). Resistance pattern of enterobacteriaceae isolates from urinary tract infections to selected quinolones in Yaounde. Pan Afr. Med. J. 21:105. doi: 10.11604/pamj.2015.21.105.5469

Magoué, C. L., Melin, P., Gangoué-Piéboji, J., Assoumou, M. C. O., Boreux, R., and De Mol, P. (2013). Prevalence and spread of extended-spectrum $\beta$-lactamase-producing Enterobacteriaceae in Ngaoundere, Cameroon. Clin. Microbiol. Infect. 19, E416-E420. doi: 10.1111/1469-0691.12239

Moussé, W., Noumavo, P. A., Chabi, N. W., Sina, H., Tohoyessou, M. G., Ahoyo, T. A., et al. (2016). Phenotypic and genotypic characterization of extended spectrum $\beta$-Lactamase Klebsiella pneumoniae and fluorescent Pseudomonas spp. strains from market garden products and their watering water in Benin (West Africa). Food Nutr. Sci. 7, 192-204. doi: 10.4236/fns.2016. 73021

Ndebi, G., Kamajou, J., and Ongla, J. (2009). Analyse des contraintes au développement de la production porcine au Cameroun. Tropicultura 27, 70-76.

Nzalie, R. N.-T., Gonsu, H. K., and Koulla-Shiro, S. (2016). Bacterial etiology and antibiotic resistance profile of community-acquired urinary tract infections in a Cameroonian city. Int. J. Microbiol. 2016:3240268. doi: 10.1155/2016/32 40268

Overbeek, R., Olson, R., Pusch, G. D., Olsen, G. J., Davis, J. J., Disz, T., et al. (2014). The SEED and the rapid annotation of microbial genomes using subsystems technology (RAST). Nucleic Acids Res. 42, D206-D214. doi: 10.1093/nar/gkt1226

Page, A. J., Cummins, C. A., Hunt, M., Wong, V. K., Reuter, S., Holden, M. T., et al. (2015). Roary: rapid large-scale prokaryote pan genome analysis. Bioinformatics 31, 3691-3693. doi: 10.1093/bioinformatics/btv421

Perovic, O., Britz, E., Chetty, V., and Singh-Moodley, A. (2016). Molecular detection of carbapenemase-producing genes in referral Enterobacteriaceae in South Africa: a short report. S. Afr. Med. J. 106, 975-977. doi: 10.7196/SAMJ. 2016.v106i10.11300

Perovic, O., Singh-Moodley, A., Duse, A., Bamford, C., Elliott, G., Swe-Han, K. S., et al. (2014). National sentinel site surveillance for antimicrobial resistance in Klebsiella pneumoniae isolates in South Africa, 2010-2012. S. Afr. Med. J. 104, 563-568. doi: 10.7196/samj.7617

Rafiq, Z., Sam, N., and Vaidyanathan, R. (2016). Whole genome sequence of Klebsiella pneumoniae U25, a hypermucoviscous, multidrug resistant, biofilm producing isolate from India. Mem. Inst. Oswaldo Cruz 111, 144-146. doi: 10.1590/0074-02760150423

The European Committee on Antimicrobial Susceptibility Testing [EUCAST]. (2016). Breakpoint Tables for Interpretation of MICs and Zone Diameters. Available at: http://www.eucast.org

Versalovic, J., Koeuth, T., and Lupski, J. R. (1991). Distribution of repetitive DNA sequences in eubacteria and application to fingerprinting of bacterial genomes. Nucleic Acids Res. 19, 6823-6831.

Villa, L., Feudi, C., Fortini, D., Brisse, S., Passet, V., Bonura, C., et al. (2017). Diversity, virulence, and antimicrobial resistance of the KPC-producing Klebsiella pneumoniae ST307 clone. Microb. Genom. 3:e000110. doi: 10.1099/ mgen.0.000110

Wachino, J., Yamane, K., Suzuki, S., Kimura, K., and Arakawa, Y. (2010). Prevalence of fosfomycin resistance among CTX-M-producing Escherichia coli clinical isolates in Japan and identification of novel plasmid-mediated fosfomycin-modifying enzymes. Antimicrob. Agents Chemother. 54, 3061-3064. doi: 10.1128/AAC.01834-09

World Health Organization [WHO] (2017). (Global) Priority List of Antibiotic Resistant Bacteria to Guide Research, Discoveries and Development of New Antibiotics. Geneva: WHO.

Xu, H., Miao, V., Kwong, W., Xia, R., and Davies, J. (2011). Identification of a novel fosfomycin resistance gene (fosA2) in Enterobacter cloacae from the Salmon River, Canada. Lett. Appl. Microbiol. 52, 427-429. doi: 10.1111/j.1472-765X. 2011.03016.x

Zankari, E., Hasman, H., Cosentino, S., Vestergaard, M., Rasmussen, S., Lund, O., et al. (2012). Identification of acquired antimicrobial resistance genes. J. Antimicrob. Chemother. 67, 2640-2644. doi: 10.1093/jac/dks261

Zhou, Y., Liang, Y., Lynch, K. H., Dennis, J. J., and Wishart, D. S. (2011). PHAST: a fast phage search tool. Nucleic Acids Res. 39(Suppl. 2), W347-W352. doi: $10.1093 /$ nar/gkr485

Conflict of Interest Statement: SE is a member of the Global Respiratory Infection Partnership sponsored by an unrestricted educational grant from Reckitt and Benckiser.

The other authors declare that the research was conducted in the absence of any commercial or financial relationships that could be construed as a potential conflict of interest.

Copyright (c) 2018 Founou, Founou, Allam, Ismail, Djoko and Essack. This is an open-access article distributed under the terms of the Creative Commons Attribution License (CC BY). The use, distribution or reproduction in other forums is permitted, provided the original author(s) and the copyright owner are credited and that the original publication in this journal is cited, in accordance with accepted academic practice. No use, distribution or reproduction is permitted which does not comply with these terms. 